\title{
THE VECTOR POTENTIAL IN ACCELERATOR MAGNETS $\uparrow$
}

\author{
C. J. GARDNER \\ Brookhaven National Laboratory, Upton, New York, 11973, USA. \\ (Received 24 July 1990; in final form 30 January 1991)
}

\begin{abstract}
The usual treatment of the vector potential in accelerator magnets assumes that the magnetic field is transverse to the reference orbit and therefore does not take into account those regions- typically near the ends of the magnets-where the field has a longitudinal component. The effects of the neglected fields in these regions are generally unimportant in high-energy particle accelerators but can become significant at lower energies in smaller accelerators or storage rings. By choosing an appropriate gauge, a complete expression for the vector potential is developed in terms of the coefficients in the general expansion of the magnetic field about the reference orbit. Applications to the end-field regions of a dipole and a quadrupole are discussed.
\end{abstract}

\section{INTRODUCTION}

The standard treatment of the static magnetic field in an accelerator involves expanding the field about the reference orbit and expressing it in terms of normal and skew multipoles, which arise in regions where the field is transverse to the reference orbit, and in terms of other non-multipole terms, which arise in regions where the field has longitudinal components or where the curvature of the reference orbit is nonzero. The coefficients in the expansion depend only on the values of the field and its derivatives on the reference orbit, and are essentially what we measure and use to characterize the various magnetic elements in an accelerator.

The coefficients also serve to specify the vector potential, which is required explicitly for some applications. However, since the vector potential is not uniquely determined by the magnetic field, its complete specification requires an appropriate gauge transformation. In regions where the magnetic field is transverse to the reference orbit - typically far inside the accelerator magnets away from the magnet ends - the choice of an appropriate gauge is straightforward. Here one usually chooses the gauge such that the vector potential has no transverse components. In this case the vector potential is completely determined except for the addition of the gradient of an arbitrary function of the position along the reference orbit which may be taken to be zero. Near the ends of the magnets the magnetic field has longitudinal components and the vector potential must therefore have transverse components. The choice of an appropriate gauge transformation in these and other regions where

$\dagger$ Work performed under the auspices of the U. S. Department of Energy. 
the magnetic field has longitudinal components is the subject of this report. It will be shown that one can choose the gauge such that the vector potential has the required transverse components near the magnet ends, has no transverse components in regions where the magnetic field is transverse to the reference orbit, and has expansion coefficients that are completely determined by the coefficients in the expansion of the magnetic field. The resulting expression for the vector potential is useful for applications in which the magnet end-fields are of interest.

\section{EXPANSION OF THE MAGNETIC FIELD ABOUT THE REFERENCE ORBIT}

Here we follow the treatment of the magnetic field given in Ref. 1. Inside the aperture of an accelerator the curl of the static magnetic field, $\mathbf{B}$, is zero which implies that B can be expressed as the gradient of a scalar potential, $\phi$. Thus

$$
\mathbf{B}=\nabla \phi,
$$

and since the divergence of $\mathbf{B}$ must also vanish we have

$$
\nabla^{2} \phi=0 .
$$

The desired expansion of the magnetic field is obtained by expanding $\phi$ about the reference orbit and then inserting this result into Eq. (1). We shall assume that the reference orbit lies in a plane and shall employ the right-handed curvilinear coordinate system $(\mathbf{x}, \mathbf{y}, \mathbf{s})$ introduced in Ref. 1. In terms of these coordinates we have

$$
\nabla \phi=\mathbf{x} \frac{\partial \phi}{\partial x}+\mathbf{y} \frac{\partial \phi}{\partial \boldsymbol{z}}+\mathbf{s}\left(\frac{1}{1+h x}\right) \frac{\partial \phi}{\partial s},
$$

and

$$
\nabla^{2} \phi=\frac{1}{1+h x} \frac{\partial}{\partial x}\left[(1+h x) \frac{\partial \phi}{\partial x}\right]+\frac{\partial^{2} \phi}{\partial y^{2}}+\frac{1}{1+h x} \frac{\partial}{\partial s}\left[\frac{1}{1+h x} \frac{\partial \phi}{\partial s}\right]
$$

where $h=h(s)$ is the curvature of the reference orbit. For each $s$ along the reference orbit we can expand the potential, $\phi$, as follows:

$$
\phi(x, y, s)=\sum_{m=0}^{\infty} \sum_{n=0}^{\infty} C_{m n} \frac{x^{n}}{n !} \frac{y^{m}}{m !}
$$

where the coefficients, $C_{m n}$, are functions of $s$. Putting this expansion into Eq. (4), we find the following recursion relation for the coefficients $C_{m n}$ :

$$
\begin{aligned}
-C_{m+2, n}= & C_{m n}^{\prime \prime}+n h C_{m, n-1}^{\prime \prime}-n h^{\prime} C_{m, n-1}^{\prime} \\
& +C_{m, n+2}+(3 n+1) h C_{m, n+1}+n(3 n-1) h^{2} C_{m n} \\
& +n(n-1)^{2} h^{3} C_{m, n-1}+3 n h C_{m+2, n-1} \\
& +3 n(n-1) h^{2} C_{m+2, n-2}+n(n-1)(n-2) h^{3} C_{m+2, n-3}
\end{aligned}
$$


where a prime denotes differentiation with respect to $s$ and all coefficients with one or more negative subscripts are, by definition, zero. Putting (5) into Eqs. (3) and (1), we find that the components of $\mathbf{B}$ in the $\mathbf{x}, \mathbf{y}$, and $\mathbf{s}$ directions are, respectively:

$$
\begin{aligned}
& B_{x}=\frac{\partial \phi}{\partial x}=\sum_{m=0}^{\infty} \sum_{n=0}^{\infty} C_{m, n+1} \frac{x^{n}}{n !} \frac{y^{m}}{m !} \\
& B_{y}=\frac{\partial \phi}{\partial y}=\sum_{m=0}^{\infty} \sum_{n=0}^{\infty} C_{m+1, n} \frac{x^{n}}{n !} \frac{y^{m}}{m !} \\
& B_{s}=\frac{1}{1+h x} \frac{\partial \phi}{\partial s}=\frac{1}{1+h x} \sum_{m=0}^{\infty} \sum_{n=0}^{\infty} C_{m, n}^{\prime} \frac{x^{n}}{n !} \frac{y^{m}}{m !} .
\end{aligned}
$$

This is the desired expansion of the magnetic field about the reference orbit.

Differentiating $B_{y}$ and $B_{x}$ with respect to $x$ we find

$$
C_{1 n}=\left(\frac{\partial^{n} B_{y}}{\partial x^{n}}\right)_{x=0, y=0}, \quad C_{10}=B_{y}(0,0, s)
$$

and

$$
C_{0, n+1}=\left(\frac{\partial^{n} B_{x}}{\partial x^{n}}\right)_{x=0, y=0}, \quad C_{01}=B_{x}(0,0, s)
$$

which are the normal and skew multipole strengths, respectively, evaluated on the reference orbit. The longitudinal component of the magnetic field on the reference orbit is given by

$$
C_{00}^{\prime}=B_{s}(0,0, s) \text {. }
$$

It follows from the recursion relation (6) that all of the coefficients in the magnetic field expansion can be expressed in terms of these multipole and longitudinal field strengths and their derivatives with respect to $s$. Equations (7) then show that the field at any point for which the expansion is valid is completely determined by the multipole and longitudinal field strengths on the reference orbit. Defining

$$
B_{n}=C_{1 n}, \quad A_{n}=C_{0, n+1}, \quad C_{0}=C_{00}^{\prime},
$$

we find that the coefficients required to expand the field to third order are given by Eqs. (8-11) and

$$
\begin{aligned}
C_{20}= & -A_{1}-h A_{0}-C_{0}^{\prime} \\
C_{21}= & -A_{2}-h A_{1}+h^{2} A_{0}-A_{0}^{\prime \prime}+2 h C_{0}^{\prime}+h^{\prime} C_{0} \\
C_{22}= & -A_{3}-h A_{2}+2 h^{2} A_{1}-2 h^{3} A_{0} \\
& -A_{1}^{\prime \prime}+4 h A_{0}^{\prime \prime}+2 h^{\prime} A_{0}^{\prime}-6 h^{2} C_{0}^{\prime}-6 h h^{\prime} C_{0} \\
C_{30}= & -B_{2}-h B_{1}-B_{0}^{\prime \prime} \\
C_{31}= & -B_{3}-h B_{2}+h^{2} B_{1}-B_{1}^{\prime \prime}+2 h B_{0}^{\prime \prime}+h^{\prime} B_{0}^{\prime} \\
C_{40}= & -C_{22}-h C_{21}-C_{20}^{\prime \prime} .
\end{aligned}
$$


The definition of the multipole coefficients, $B_{n}$, given in Eqs. (11) and (8) is consistent with that used in the MAD program ${ }^{2}$.

\section{EXPANSION OF THE VECTOR POTENTIAL ABOUT THE REFERENCE ORBIT}

Since its divergence vanishes, the magnetic field can be expressed as the curl of a vector potential, A. Thus

$$
\mathbf{B}=\nabla \times \mathbf{A},
$$

which, in terms of the curvilinear coordinate system introduced in Ref. 1, becomes

$$
\begin{aligned}
B_{x} & =\frac{\partial A_{s}}{\partial y}-\frac{1}{1+h x} \frac{\partial A_{y}}{\partial s} \\
B_{y} & =\frac{1}{1+h x}\left(\frac{\partial A_{x}}{\partial s}-h A_{s}\right)-\frac{\partial A_{s}}{\partial x} \\
B_{s} & =\frac{\partial A_{y}}{\partial x}-\frac{\partial A_{x}}{\partial y} .
\end{aligned}
$$

Here, as before, the subscripts $x, y$, and $s$ denote the components of the vectors in the $\mathbf{x}, \mathbf{y}$, and $\mathbf{s}$ directions. Expanding $A_{x}, A_{y}$, and $A_{s}$ about the reference orbit, we have

$$
\begin{aligned}
& A_{x}=\sum_{m=0}^{\infty} \sum_{n=0}^{\infty} a_{m n} \frac{x^{n}}{n !} \frac{y^{m}}{m !} \\
& A_{y}=\sum_{m=0}^{\infty} \sum_{n=0}^{\infty} b_{m n} \frac{x^{n}}{n !} \frac{y^{m}}{m !} \\
& A_{s}=\sum_{m=0}^{\infty} \sum_{n=0}^{\infty} d_{m n} \frac{x^{n}}{n !} \frac{y^{m}}{m !},
\end{aligned}
$$

where $a_{m n}, b_{m n}$, and $d_{m n}$ are functions of $s$. Inserting Eq. (15) into Eq. (14) and comparing with Eq. (7), we find the following relations between the magnetic field and vector potential expansion coefficients:

$$
\begin{gathered}
C_{m, n+1}+n h C_{m n}=d_{m+1, n}-b_{m n}^{\prime}+n h d_{m+1, n-1} \\
C_{m+1, n}+n h C_{m+1, n-1}=a_{m n}^{\prime}-d_{m, n+1}-(1+n) h d_{m n} \\
C_{m n}^{\prime}=b_{m, n+1}-a_{m+1, n}+n h b_{m n}-n h a_{m+1, n-1} .
\end{gathered}
$$

These relations, together with an appropriate gauge transformation, will completely specify the vector potential. 


\section{GAUGE TRANSFORMATION}

Since we can add the gradient of a scalar function, $f$, to the vector potential, A, without changing the magnetic field, the general expansion of $\mathbf{A}$ about the reference orbit is of the form

$$
\mathbf{A}=\mathbf{V}+\nabla f
$$

where

$$
\begin{aligned}
V_{x} & =\sum_{m=0}^{\infty} \sum_{n=0}^{\infty} u_{m n} \frac{x^{n}}{n !} \frac{y^{m}}{m !} \\
V_{y} & =\sum_{m=0}^{\infty} \sum_{n=0}^{\infty} v_{m n} \frac{x^{n}}{n !} \frac{y^{m}}{m !} \\
V_{s} & =\sum_{m=0}^{\infty} \sum_{n=0}^{\infty} w_{m n} \frac{x^{n}}{n !} \frac{y^{m}}{m !}
\end{aligned}
$$

and

$$
f=\sum_{m=0}^{\infty} \sum_{m=0}^{\infty} f_{m n} \frac{x^{n}}{n !} \frac{y^{m}}{m !}
$$

Thus, using Eqs. (18) and (19) in Eq. (17) and comparing with Eq. (15), we find

$$
\begin{aligned}
a_{m n} & =u_{m n}+f_{m, n+1} \\
b_{m n} & =v_{m n}+f_{m+1, n} \\
d_{m n}+n h d_{m, n-1} & =w_{m n}+n h w_{m, n-1}+f_{m n}^{\prime} .
\end{aligned}
$$

Now, since $f$ is arbitrary we may choose

$$
f_{m, n+1}=-u_{m n}, \quad f_{m+1,0}=-v_{m 0}, \quad f_{00}^{\prime}=-w_{00},
$$

in which case we have

$$
a_{m n}=0, \quad b_{m 0}=0, \quad d_{00}=0,
$$

and Eqs. (16) become

$$
\begin{aligned}
C_{m, n+1}+n h C_{m n} & =d_{m+1, n}-b_{m n}^{\prime}+n h d_{m+1, n-1} \\
C_{m+1, n}+n h C_{m+1, n-1} & =-d_{m, n+1}-(1+n) h d_{m n} \\
C_{m n}^{\prime} & =b_{m, n+1}+n h b_{m n} .
\end{aligned}
$$

These relations completely determine the nonzero coefficients $b_{m n}$ and $d_{m n}$. Solving Eq. (23), one obtains these coefficients entirely in terms of the coefficients, $C_{m n}$, which in turn can be expressed in terms of the multipole and longitudinal field strengths 
defined by Eqs. (8-11). Using Eqs. (11-12) and (22) in Eq. (23), we find

$$
\begin{aligned}
d_{01}= & -B_{0} \\
d_{02}= & -B_{1}+h B_{0} \\
d_{03}= & -B_{2}+h B_{1}-3 h^{2} B_{0} \\
d_{04}= & -B_{3}+h B_{2}-4 h^{2} B_{1}+12 h^{3} B_{0} \\
d_{10}= & A_{0} \\
d_{11}= & A_{1}+C_{0}^{\prime} \\
d_{12}= & A_{2}+A_{0}^{\prime \prime}-3 h C_{0}^{\prime}-h^{\prime} C_{0} \\
d_{13}= & A_{3}+A_{1}^{\prime \prime}-5 h A_{0}^{\prime \prime}-2 h^{\prime} A_{0}^{\prime}+11 h^{2} C_{0}^{\prime}+7 h h^{\prime} C_{0} \\
d_{20}= & B_{1} \\
d_{21}= & B_{2}+B_{0}^{\prime \prime} \\
d_{22}= & B_{3}+B_{1}^{\prime \prime}-3 h B_{0}^{\prime \prime}-h^{\prime} B_{0}^{\prime} \\
d_{30}= & -A_{2}-h A_{1}+h^{2} A_{0}-A_{0}^{\prime \prime}+2 h C_{0}^{\prime}+h^{\prime} C_{0} \\
d_{31}= & -A_{3}-h A_{2}+2 h^{2} A_{1}-2 h^{3} A_{0} \\
& -2 A_{1}^{\prime \prime}+3 h A_{0}^{\prime \prime}-h^{\prime \prime} A_{0}-C_{0}^{\prime \prime}-6 h^{2} C_{0}^{\prime}-6 h h^{\prime} C_{0} \\
d_{40}= & -B_{3}-h B_{2}+h^{2} B_{1}-B_{1}^{\prime \prime}+2 h B_{0}^{\prime \prime}+h^{\prime} B_{0}^{\prime},
\end{aligned}
$$

and

$$
\begin{aligned}
& b_{01}=C_{0} \\
& b_{02}=A_{0}^{\prime}-h C_{0} \\
& b_{03}=A_{1}^{\prime}-2 h A_{0}^{\prime}+2 h^{2} C_{0} \\
& b_{04}=A_{2}^{\prime}-3 h A_{1}^{\prime}+6 h^{2} A_{0}^{\prime}-6 h^{3} C_{0} \\
& b_{11}=B_{0}^{\prime} \\
& b_{12}=B_{1}^{\prime}-h B_{0}^{\prime} \\
& b_{13}=B_{2}^{\prime}-2 h B_{1}^{\prime}+2 h^{2} B_{0}^{\prime} \\
& b_{21}=-A_{1}^{\prime}-h^{\prime} A_{0}-h A_{0}^{\prime}-C_{0}^{\prime \prime} \\
& b_{22}=-A_{2}^{\prime}-h^{\prime} A_{1}+3 h h^{\prime} A_{0}+2 h^{2} A_{0}^{\prime}-A_{0}^{\prime \prime \prime}+3 h^{\prime} C_{0}^{\prime}+3 h C_{0}^{\prime \prime}+h^{\prime \prime} C_{0} \\
& b_{31}=-B_{2}^{\prime}-h^{\prime} B_{1}-h B_{1}^{\prime}-B_{0}^{\prime \prime \prime} .
\end{aligned}
$$

Continuing in this way one obtains all of the coefficients $b_{m n}$ and $d_{m n}$ in terms of the multipole and longitudinal field strengths and their derivatives with respect to $s$. For the case in which the magnetic field is transverse to the reference orbit, the coefficients $C_{m n}^{\prime}$ are zero and it follows from Eq. (22) and the last of Eqs. (23) that the coefficients $b_{m n}$ are all zero. The vector potential then has only the longitudinal 
component, $A_{s}$. In regions where the coefficients $C_{m n}^{\prime}$ are nonzero the magnetic field has a longitudinal component, the coefficients $b_{m n}$ are nonzero, and the vector potential has a transverse component, $A_{y}$.

The components of the vector potential to fourth order can be obtained by inserting Eqs. (22) and (24-25) into Eq. (15). Collecting terms we find

$$
A_{s}=A_{s}^{a}+A_{s}^{b} \text {, }
$$

where (to fourth order)

$$
\begin{aligned}
A_{s}^{a}= & -B_{0}\left[x-h x^{2} / 2+h^{2} x^{3} / 2-h^{3} x^{4} / 2\right] \\
& -B_{1}\left[\left(x^{2}-y^{2}\right) / 2-h x^{3} / 6+h^{2}\left(4 x^{4}-y^{4}\right) / 24\right] \\
& -B_{2}\left[\left(x^{3}-3 x y^{2}\right) / 6-h\left(x^{4}-y^{4}\right) / 24\right] \\
& -B_{3}\left[\left(x^{4}-6 x^{2} y^{2}+y^{4}\right) / 24\right] \\
& -A_{0}\left[-y-h^{2} y^{3} / 6+h^{3} x y^{3} / 3\right] \\
& -A_{1}\left[-x y+h y^{3} / 6-h^{2} x y^{3} / 3\right] \\
& -A_{2}\left[\left(y^{3}-3 x^{2} y\right) / 6+h x y^{3} / 6\right] \\
& -A_{3}\left[\left(x y^{3}-x^{3} y\right) / 6\right],
\end{aligned}
$$

and

$$
\begin{aligned}
A_{s}^{b}= & -B_{0}^{\prime \prime}\left[-x y^{2} / 2+h\left(9 x^{2} y^{2}-y^{4}\right) / 12\right]-B_{0}^{\prime} h^{\prime}\left[\left(6 x^{2} y^{2}-y^{4}\right) / 24\right] \\
& -B_{1}^{\prime \prime}\left[\left(y^{4}-6 x^{2} y^{2}\right) / 24\right] \\
& -A_{0}^{\prime \prime}\left[\left(y^{3}-3 x^{2} y\right) / 6+h\left(5 x^{3} y-3 x y^{3}\right) / 6\right] \\
& -A_{0}^{\prime} h^{\prime}\left[x^{3} y / 3\right]-A_{0} h^{\prime \prime}\left[x y^{3} / 6\right] \\
& -A_{1}^{\prime \prime}\left[\left(2 x y^{3}-x^{3} y\right) / 6\right] \\
& -C_{0}^{\prime}\left[-x y-h\left(2 y^{3}-9 x^{2} y\right) / 6+h^{2}\left(6 x y^{3}-11 x^{3} y\right) / 6\right] \\
& -C_{0} h^{\prime}\left[\left(3 x^{2} y-y^{3}\right) / 6+h\left(6 x y^{3}-7 x^{3} y\right) / 6\right]-C_{0}^{\prime \prime \prime}\left[x y^{3} / 6\right],
\end{aligned}
$$

and

$$
\begin{aligned}
A_{y}= & h^{\prime} A_{0}\left[-x y^{2} / 2+3 h x^{2} y^{2} / 4\right]-A_{0}^{\prime \prime \prime}\left[x^{2} y^{2} / 4\right] \\
& +A_{0}^{\prime}\left[x^{2} / 2-h\left(2 x^{3}+3 x y^{2}\right) / 6+h^{2}\left(x^{4}+2 x^{2} y^{2}\right) / 4\right] \\
& +A_{1}^{\prime}\left[\left(x^{3}-3 x y^{2}\right) / 6-h x^{4} / 8\right]-h^{\prime} A_{1}\left[x^{2} y^{2} / 4\right] \\
& +A_{2}^{\prime}\left[\left(x^{4}-6 x^{2} y^{2}\right) / 24\right] \\
& +B_{0}^{\prime}\left[x y-h x^{2} y / 2+2 h^{2} x^{3} y / 6\right]-B_{0}^{\prime \prime \prime}\left[x y^{3} / 6\right] \\
& +B_{1}^{\prime}\left[x^{2} y / 2-h\left(2 x^{3} y+x y^{3}\right) / 6\right]-h^{\prime} B_{1}\left[x y^{3} / 6\right] \\
& +B_{2}^{\prime}\left[\left(x^{3} y-x y^{3}\right) / 6\right] \\
& +C_{0}^{\prime}\left[3 h^{\prime} x^{2} y^{2} / 4\right]+C_{0}^{\prime \prime}\left[-x y^{2} / 2+3 h x^{2} y^{2} / 4\right] \\
& +C_{0}\left[x-h x^{2} / 2+2 h^{2} x^{3} / 6-6 h^{3} x^{4} / 24+h^{\prime \prime} x^{2} y^{2} / 4\right] .
\end{aligned}
$$


Equations (27-29) show explicitly that in the regions where $C_{0}(s)=0$ and the multipole strengths and curvature are independent of $s$, the vector potential has only a longitudinal component and is given by $A_{s}^{a}$. Outside these regions, and in particular near the magnet ends, the vector potential has the additional terms given by $A_{s}^{b}$ and $A_{y}$. The expression obtained for $A_{s}^{a}$ is consistent with that given in Ref. 2 , and contains only pure multipole terms for the case in which the curvature, $h$, is zero.

We have shown that the gauge specified by Eqs. (21-22) allows for a complete determination of the vector potential in terms of the coefficients in the expansion of the magnetic field about the reference orbit. This is not, of course, the only gauge one may choose, but it is a rather simple one. An example of another gauge, which treats the $x$ and $y$ components of the vector potential symmetrically, is discussed in the Appendix.

\section{APPLICATIONS}

The equations of motion for betatron oscillations about the reference orbit are derived from the Hamiltonian ${ }^{3}$

$$
H=-a P_{T}-(1+h x)\left[1-2 a P_{T}+P_{T}^{2}-\left(P_{x}-U\right)^{2}-\left(P_{y}-V\right)^{2}\right]^{1 / 2}-(1+h x) W,
$$

where

$$
U=e A_{x} /\left(c p_{0}\right), \quad V=e A_{y} /\left(c p_{0}\right), \quad W=e A_{s} /\left(c p_{0}\right), \quad P_{x}=p_{x} / p_{0}, \quad P_{y}=p_{y} / p_{0},
$$

$A_{x}, A_{y}, A_{s}$ are the components of the vector potential, $p_{x}$ and $p_{y}$ are the momentum components along $x$ and $y$, and $p_{0}=m c \beta_{0} \gamma_{0}$ is the momentum of the reference particle. The parameter, $a$, depends on the choice of the momentum variable $P_{T}$. If $P_{T}$ is taken to be the negative momentum deviation divided by $p_{0}$ (i.e., $P_{T}=$ $\left.-\left(p-p_{0}\right) / p_{0}\right)$ then $a=1$. Alternatively, if $P_{T}$ is taken to be the negative energy deviation divided by $c p_{0}$ (i.e., $P_{T}=-\left(E-E_{0}\right) /\left(c p_{0}\right)$ then $a=1 / \beta_{0}$. Expanding $H$ to 4 th order in the coordinates and momenta, we obtain

$$
H=H_{0}+H_{1}+H_{2}+H_{3}+H_{4}
$$

where

$$
\begin{aligned}
H_{1}= & -\left(W_{1}+h x\right), \\
H_{2}= & \left(P_{x}^{2}+P_{y}^{2}\right) / 2-W_{2}-h x W_{1}+a h x P_{T} \\
& +\left(U_{1}^{2}+V_{1}^{2}\right) / 2-\left(U_{1} P_{x}+V_{1} P_{y}\right)+\left(a^{2}-1\right) P_{T}^{2} / 2, \\
H_{3}= & -W_{3}-h x W_{2}+\left(U_{1} U_{2}+V_{1} V_{2}\right)-\left(U_{2} P_{x}+V_{2} P_{y}\right) \\
& +\left[P_{x}^{2}+P_{y}^{2}-2\left(U_{1} P_{x}+V_{1} P_{y}\right)+U_{1}^{2}+V_{1}^{2}\right] h x / 2 \\
& +\left[P_{x}^{2}+P_{y}^{2}-2\left(U_{1} P_{x}+V_{1} P_{y}\right)+U_{1}^{2}+V_{1}^{2}\right] a P_{T} / 2 \\
& +a\left(a^{2}-1\right) P_{T}^{3} / 2+\left(a^{2}-1\right) h x P_{T}^{2} / 2,
\end{aligned}
$$




$$
\begin{aligned}
H_{4}= & \left(P_{x}^{2}+P_{y}^{2}\right)^{2} / 8-\left(P_{x}^{2}+P_{y}^{2}\right)\left(U_{1} P_{x}+V_{1} P_{y}\right) / 2+\left(U_{1} P_{x}+V_{1} P_{y}\right)^{2} / 2 \\
& +\left(P_{x}^{2}+P_{y}^{2}\right)\left(U_{1}^{2}+V_{1}^{2}\right) / 4-\left(U_{1} P_{x}+V_{1} P_{y}\right)\left(U_{1}^{2}+V_{1}^{2}\right) / 2+\left(U_{1}^{2}+V_{1}^{2}\right)^{2} / 8 \\
& -\left(U_{3} P_{x}+V_{3} P_{y}\right)+\left(U_{1} U_{3}+V_{1} V_{3}\right)+\left(U_{2}^{2}+V_{2}^{2}\right) / 2 \\
& -W_{4}-h x W_{3}-h x\left(U_{2} P_{x}+V_{2} P_{y}\right)+h x\left(U_{1} U_{2}+V_{1} V_{2}\right) \\
& +\left[P_{x}^{2}+P_{y}^{2}-2\left(U_{1} P_{x}+V_{1} P_{y}\right)+U_{1}^{2}+V_{1}^{2}\right]\left(3 a^{2}-1\right) P_{T}^{2} / 4 \\
& -a P_{T}\left(U_{2} P_{x}+V_{2} P_{y}\right)+a P_{T}\left(U_{1} U_{2}+V_{1} V_{2}\right) \\
& +h x\left[P_{x}^{2}+P_{y}^{2}-2\left(U_{1} P_{x}+V_{1} P_{y}\right)+U_{1}^{2}+V_{1}^{2}\right] a P_{T} / 2 \\
& +a\left(a^{2}-1\right) h x P_{T}^{3} / 2+\left(5 a^{2}-1\right)\left(a^{2}-1\right) P_{T}^{4} / 8,
\end{aligned}
$$

and $U_{n}, V_{n}, W_{n}$ are the $n$th order terms of $U, V$, and $W$. (We have omitted the term $H_{0}$, which has no effect on the equations of motion.)

In the following discussion we consider the end-field regions of a dipole and a quadrupole. The equations of motion in these regions can, of course, be derived directly from the Lorentz force equation without explicit use of the vector potential; however, for some applications, a Hamiltonian formulation is preferable, and it is for these cases that the complete expansion of the vector potential has been developed. We consider first the case of the quadrupole, which is simpler because the curvature of the reference orbit along its axis is zero. This is followed by the more complicated case of the dipole, where the curvature and its derivatives are nonzero.

\subsection{The Quadrupole}

The scalar potential and magnetic field for a magnet with quadrupole symmetry are given in Refs. 4 and 5. Since $\phi(x, y, s)$ is an odd function of both $x$ and $y$ for such a magnet, we have (to fourth order)

$$
\phi=B_{1} x y+B_{3} x^{3} y / 6-\left(B_{3}+B_{1}^{\prime \prime}\right) x y^{3} / 6
$$

with the constraint that

$$
B_{3}=-B_{1}^{\prime \prime} / 2 \text {, }
$$

which follows from the additional requirement that $\phi(x, y, s)=\phi(y, x, s)$ for a pure quadrupole. The vector potential is then

$$
\begin{aligned}
& A_{s}=-B_{1}\left(x^{2}-y^{2}\right) / 2+B_{1}^{\prime \prime}\left(x^{4}+6 x^{2} y^{2}-y^{4}\right) / 48 \\
& A_{y}=B_{1}^{\prime} x^{2} y / 2
\end{aligned}
$$

and the components of the magnetic field, obtained from Eqs. (1) and (3) or from Eqs. (14), are

$$
\begin{aligned}
& B_{x}=B_{1} y-B_{1}^{\prime \prime}\left(3 x^{2} y+y^{3}\right) / 12 \\
& B_{y}=B_{1} x-B_{1}^{\prime \prime}\left(x^{3}+3 x y^{2}\right) / 12 \\
& B_{s}=B_{1}^{\prime} x y .
\end{aligned}
$$


Using Eqs. (39) in Eqs. (31-36), with $A_{x}=0$ and $h=0$, we obtain the Hamiltonian for motion in the quadrupole to fourth order:

where

$$
H=H_{2}+H_{3}+H_{4},
$$

$$
\begin{aligned}
H_{2}= & \left(P_{x}^{2}+P_{y}^{2}\right) / 2+K_{1}\left(x^{2}-y^{2}\right) / 2+\left(a^{2}-1\right) P_{T}^{2} / 2, \\
H_{3}= & a P_{T}\left(P_{x}^{2}+P_{y}^{2}\right) / 2+a\left(a^{2}-1\right) P_{T}^{3} / 2, \\
H_{4}= & \left(P_{x}^{2}+P_{y}^{2}\right)^{2} / 8-K_{1}^{\prime} x^{2} y P_{y} / 2-K_{1}^{\prime \prime}\left(x^{4}+6 x^{2} y^{2}-y^{4}\right) / 48 \\
& +\left(3 a^{2}-1\right) P_{T}^{2}\left(P_{x}^{2}+P_{y}^{2}\right) / 4+\left(5 a^{2}-1\right)\left(a^{2}-1\right) P_{T}^{4} / 8,
\end{aligned}
$$

and $K_{1}=e B_{1} /\left(c p_{0}\right)$. Hamilton's equations then yield equations of motion to third order that agree with those obtained directly from the Lorentz force equation by Steffen ${ }^{4}$ and Carey ${ }^{5}$. Lee-Whiting ${ }^{6}$ has solved these equations to obtain expressions for the third-order transfer map through the end-field region. An approximate analysis by Carey ${ }^{5}$ shows that the effects of the end-fields are generally negligible in high-energy accelerators, but can become important for lower energies and larger apertures.

Lie algebraic techniques ${ }^{3,7}$, which employ the Hamiltonian directly, can also be used to obtain the transfer map. The second, third, and fourth order Lie algebraic polynomials associated with the transfer map are obtained from $\mathrm{H}_{2}, \mathrm{H}_{3}$, and $\mathrm{H}_{4}$ as shown in Ref. 7. Ryne and Dragt ${ }^{8}$ have used these methods to obtain the third-order transfer map for motion through the end-field regions of the quadrupole.

\subsection{The Dipole}

In the end-field regions of a dipole magnet with midplane symmetry, the curvature of the reference trajectory is not constant and the expansion of the magnetic field about the reference trajectory will generally contain all multipole components allowed for a magnet with midplane symmetry. The scalar potential for such a magnet is an odd function of $y$. This implies that all terms in Eqs. (26-29) containing $A_{n}, C_{0}$, or their derivatives are zero. Substitution of the resulting vector potential into Eqs. (31-36) yields the Hamiltonian to fourth order:

where

$$
\mathrm{H}=\mathrm{H}_{2}+\mathrm{H}_{3}+\mathrm{H}_{4} \text {, }
$$

$$
\begin{aligned}
H_{2}= & \left(P_{x}^{2}+P_{y}^{2}\right) / 2+h^{2} x^{2} / 2+K_{1}\left(x^{2}-y^{2}\right) / 2+a h x P_{T}+\left(a^{2}-1\right) P_{T}^{2} / 2 \\
H_{3}= & K_{2}\left(x^{3}-3 x y^{2}\right) / 6+h K_{1}\left(2 x^{3}-3 x y^{2}\right) / 6-h^{\prime \prime} x y^{2} / 2-h^{\prime} x y P_{y} \\
& +h x\left(P_{x}^{2}+P_{y}^{2}\right) / 2+a P_{T}\left(P_{x}^{2}+P_{y}^{2}\right) / 2+a\left(a^{2}-1\right) P_{T}^{3} / 2+\left(a^{2}-1\right) h x P_{T}^{2} / 2 \\
H_{4}= & \left(P_{x}^{2}+P_{y}^{2}\right)^{2} / 8+K_{3}\left(x^{4}-6 x^{2} y^{2}+y^{4}\right) / 24+h K_{2}\left(3 x^{4}-12 x^{2} y^{2}+y^{4}\right) / 24 \\
& -h^{2} K_{1} y^{4} / 24-h^{\prime 2} y^{4} / 24+3 h^{\prime 2} x^{2} y^{2} / 4-h h^{\prime} x^{2} y P_{y} / 2-K_{1}^{\prime} x^{2} y P_{y} / 2 \\
& +h h^{\prime \prime}\left(3 x^{2} y^{2}-y^{4}\right) / 12+K_{1}^{\prime \prime}\left(y^{4}-6 x^{2} y^{2}\right) / 24-a h^{\prime} x y P_{y} P_{T} \\
& +a h x\left(P_{x}^{2}+P_{y}^{2}\right) P_{T} / 2+\left(3 a^{2}-1\right) P_{T}^{2}\left(P_{x}^{2}+P_{y}^{2}\right) / 4 \\
& +a\left(a^{2}-1\right) h x P_{T}^{3} / 2+\left(5 a^{2}-1\right)\left(a^{2}-1\right) P_{T}^{4} / 8,
\end{aligned}
$$


$h=e B_{0} /\left(c p_{0}\right), K_{n}=e B_{n} /\left(c p_{0}\right)$, and $p_{0}$ is the reference momentum. Sufficiently far inside the dipole, the magnetic field is independent of $x, y$, and $s$, and the Hamiltonian is given by Eqs. (43-44) with the terms involving derivatives with respect to $s$ and those involving $K_{1}, K_{2}$, and $K_{3}$ set equal to zero.

The transfer map for motion through the end-fields of the dipole has been calculated by Lee-Whiting ${ }^{9}$ and Sagalovsky ${ }^{10}$. Lee-Whiting obtains expressions for the transfer map to second order in terms of various integrals over the end-field region. Sagalovsky obtains expressions for the map to third order in terms of power series expansions in the parameter $\varepsilon=d / \rho$, where $d$ is the vertical separation of the magnet poles and $\rho$ is the radius of curvature inside the dipole away from the end-field region. As with the quadrupole, one can also obtain the transfer map using Lie algebraic techniques. In this case the reference trajectory must first be found by numerical integration of the equations of motion. The second, third, and fourth order Lie algebraic polynomials associated with the transfer map are then obtained from $\mathrm{H}_{2}, \mathrm{H}_{3}$, and $\mathrm{H}_{4}$ as shown in Ref. 7. Ryne and Dragt ${ }^{8}$ have employed these techniques to obtain the third-order transfer map for motion through the end-field regions of the dipole.

\section{APPENDIX}

Here we consider a gauge which treats the $x$ and $y$ components of the vector potential symmetrically. If, instead of Eqs. (21), we choose

$$
\begin{aligned}
f_{m+1, n+1} & =-\left(u_{m+1, n}+v_{m, n+1}\right) / 2, \quad f_{m+1,0}=-v_{m 0}, \quad f_{0, n+1}=-u_{0 n}, \\
f_{00}^{\prime} & =-w_{00}
\end{aligned}
$$

then we have

$$
b_{m, n+1}=-a_{m+1, n}, \quad b_{m 0}=0, \quad a_{0 n}=0, \quad d_{00}=0,
$$

and Eqs. (16) become

$$
\begin{aligned}
C_{m, n+1}+n h C_{m n} & =d_{m+1, n}-b_{m n}^{\prime}+n h d_{m+1, n-1} \\
C_{m+1, n}+n h C_{m+1, n-1} & =-b_{m-1, n+1}^{\prime}-d_{m, n+1}-(1+n) h d_{m n} \\
C_{m n}^{\prime} & =2\left(b_{m, n+1}+n h b_{m n}\right) .
\end{aligned}
$$

As before, these relations completely determine the vector potential.

It follows from the first of Eqs. (34) that

$$
\frac{\partial A_{y}}{\partial x}=-\frac{\partial A_{x}}{\partial y}
$$

and hence the two transverse components of the vector potential contribute equally to the longitudinal component of the magnetic field in the last of Eqs. (14). 


\section{REFERENCES}

1. K. L. Brown and R. V. Servranckx, "First- and Second-Order Charged Particle Optics", Physics of High Energy Particle Accelerators, AIP Conference Proceedings No. 127, American Institute of Physics, New York, 1985, pp. 62-138.

2. F. C. Iselin and J. Niederer, CERN/LEP-TH/88-38, Geneva, July 13, 1988, p. 1.5 .

3. A. J. Dragt, "Lectures on Nonlinear Orbit Dynamics", Physics of High Energy Particle Accelerators, AIP Conference Proceedings No. 87, American Institute of Physics, New York, 1982, pp. 147-313.

4. K. G. Steffen, High Energy Beam Optics, Interscience Publishers, New York, 1965, pp. 47-62.

5. D. C. Carey, The Optics of Charged Particle Beams, Harwood Academic Publishers, New York, 1987, p. $148-158$.

6. G. E. Lee-Whiting, Nucl. Instrum. Meth. 83, 232 (1970).

7. A. J. Dragt and E. Forest, J. Math. Phys. 24, 2734 (1983).

8. R. D. Ryne and A. J. Dragt, Proceedings of the 1987 IEEE Particle Accelerator Conference, CH2387-9/87/0000-1081.

9. G. E. Lee-Whiting, Nucl. Instrum. Meth. A 294, 31 (1990).

10. L. Sagalovsky, Nucl. Instrum. Meth. A 298, 205 (1990). 